maximum value of $29.3 \%$. For the very low birth weight indicator, the average minimum was $1.15 \%$ and average maximum $3.88 \%$ (2.32 times the published upper confidence interval).

Discussion There is substantial geographical variation in the proportion of patients opting out of sharing their NHS data for research and planning. As shown by this simulation, even in areas with average rates of opt outs, published health indicators may be biased and not reflect the true picture of health outcomes. This is particularly the case for rarer - and potentially more severe - events. It is essential that patients are informed of the implications of opting out when deciding whether to consent to sharing their health data for research and planning purposes.

\section{RF16 THE EFFECTS OF CARING FOR YOUNG DISABLED CHILDREN ON MOTHERS' HEALTH AND HEALTHCARE USE: FINDINGS FROM THE BORN IN BRADFORD COHORT STUDY}

SC Masefield*, SL Prady, KE Pickett. Health Sciences, University of York, York, UK

\subsection{6/jech-2019-SSMabstracts. 131}

Rationale Mothers of older disabled children have worse health than mothers of typically developing children but may visit the GP less about their health problems. It is unknown whether these disparities are also present during the preschool period (0-5 years). This study compared the prevalence and frequency of GP visits for symptoms of maternal ill-health in mothers of preschool children with developmental disabilities compared with other mothers.

Methods The primary care records of children in the Born in Bradford cohort study were searched for developmental disability diagnoses before the age of five. Mothers of these children were considered exposed $(n=477)$; all other mothers unexposed $(n=9,250)$. Bivariate and multivariate logistic regression reporting odds ratios (OR) was used to compare the prevalence of psychological distress, head and musculoskeletal (MSK) pain, and exhaustion for exposed versus unexposed mothers in the year prior and five years after the child's birth. Bivariate and multivariate zero-inflated negative binomial regression reporting relative rate ratios (RRR) was used to compare GP visit frequency for these symptoms. Covariates were mother's age, ethnicity, education, subjective financial status, cohabitation status and pre-natal data (in the post-natal models).

Results Prevalence varied little pre-birth. Exposed mothers were more likely than unexposed mothers to have post-natal psychological distress $(34.6 \%$ exposed versus $30.2 \%$ unexposed; OR $1.23 ; 95 \%$ CI $1.00,1.52)$ and exhaustion $(19.3 \%$ versus $14.4 \% ; 1.41 ; 1.11,1.78)$ after the child's birth, but not head and MSK pain $(43.4 \%$ versus $39.1 \%$; 1.17 ; 0.96,1.42). Adjusting for covariates did not attenuate these findings. Visit frequency also varied little pre-birth. Visits to the GP increased after the birth, and exposed mothers did not consult more frequently than unexposed: psychological distress (mean 1.1 (standard deviation 2.0) exposed versus 0.9 (2.3) unexposed; RRR 1.00; 95\% confidence interval 0.83 , 1.20); head and MSK pain (1.0 (1.7) versus 0.9 (1.7); 1.08; $0.83,1.40)$; exhaustion $(0.3(0.6)$ versus $0.2(0.5) ; 1.19 ; 0.76$, 1.85). Pakistani ethnicity (versus white British) and pre-natal consultation were strongly associated with higher post-natal consultation rates.
Conclusion Mothers of young children with developmental disabilities may have an increased risk of some symptoms of ill health, but do not consult more frequently. The provision of early family-centred support and increased GP awareness of caregiver status could help identify and support those at risk.

\section{RF17 THE EFFECT OF ACCELEROMETER-BASED SLEEP TRAITS ON OBSTERIC OUTCOMES: A MENDELIAN RANDOMIZATION STUDY USING UK BIOBANK}

${ }^{1,2} \mathrm{Q}$ Yang* ${ }^{*}{ }^{1,2} \mathrm{MC}$ Borges, ${ }^{1,2} \mathrm{E}$ Sanderson, ${ }^{1,2,3} \mathrm{~K}$ Tilling, ${ }^{1,2,3} \mathrm{DA}$ Lawlor. ${ }^{1} \mathrm{MRC}$ Integrative Epidemiology Unit, University of Bristol, Bristol, UK; ${ }^{2}$ Population Health Sciences, Bristol Medical School, University of Bristol, Bristol, UK; ${ }^{3}$ NIHR Bristol Biomedical Centre, University Hospitals Bristol NHS Foundation Trust and University of Bristol, Bristol, UK

\subsection{6/jech-2019-SSMabstracts. 132}

Background Poor sleep is associated with increased risks of adverse pregnancy and perinatal outcomes in some studies using multivariable analyses, but these associations may be due to confounding, or biased due to the use of self-reported sleep measurements. Mendelian randomization (MR) is less vulnerable to confounding than multivariable regression and using accelerometers to objectively assess sleep will not be affected by reporting bias. Our aim was to use MR to assess the impact of four accelerometer-based sleep traits (i.e. duration, efficiency, fragmentation and chronotype) on obstetric outcomes (pregnancy loss, gestational diabetes, postnatal depression and offspring birthweight).

Methods We conducted a two-sample MR in UK Biobank (UKB). Genetic variants were selected as those that were genome-wide significant in a previous genome-wide association study completed in a sub-sample of UKB females and males $(\mathrm{N}=85,670)$ : sleep duration (11 variants), efficiency (5 variants), fragmentation (21 variants) and chronotype (6 variants). We estimated the association of each variant with self-reported obstetric outcomes among UKB female of European descent ( $N=30,310-265,680$ for different outcomes with smaller $\mathrm{Ns}$ for data available only in online follow-up). In main analyses, we used inverse variance weighting (IVW) to estimate the influence of sleep traits on each outcome. Sensitivity analyses to explore instrument validity included MR-Egger, weighted median and weighted mode estimators. All analyses were performed using R.

Results In IVW analyses, an hour increase in sleep duration was associated with $45 \mathrm{~g}$ (95\% confidence interval (CI): -80, 10) lower birthweight, but little evidence was found for effects on any pregnancy loss (odds ratio (OR): 0.97; 95\%CI: 0.86, 1.10), stillbirth (OR: 1.24; 95\%CI: 0.82, 1.87), miscarriage (OR: $0.92 ; 95 \% \mathrm{CI}: 0.80,1.07)$, gestational diabetes (OR: $0.94 ; 95 \%$ CI: $0.35,2.55)$, postnatal depression (OR: 1.18; 95\% CI: $0.79,1.77)$. We also found little evidence for an effect of sleep efficiency, fragmentation and chronotype on any of the obstetric outcomes considered. Results of sensitivity analyses were largely consistent with IVW analyses. Estimates for sleep duration-birthweight effect were -42 g (95\% CI: -90, $5)$ in weighted median, $-23 \mathrm{~g}(95 \% \mathrm{CI}:-84,39)$ in weighted mode and $18 \mathrm{~g}(95 \% \mathrm{CI}:-74,110)$ in MR-Egger.

Conclusion We did not find consistent evidence supporting an effect of poor sleep on obstetric outcomes. However, even with large sample sizes some of our results are imprecisely estimated and have wide CIs, and we did not have data to test the relevance of our instruments during pregnancy and to 